\title{
Genome-wide QTL and eQTL analyses using Mendel
}

\author{
Hua Zhou ${ }^{1 *}$, Jin Zhou ${ }^{3}$, Tao Hu ${ }^{1,2}$, Eric M. Sobel ${ }^{4}$ and Kenneth Lange ${ }^{4,5,6}$
}

From Genetic Analysis Workshop 19

Vienna, Austria. 24-26 August 2014

\begin{abstract}
Pedigree genome-wide association studies (GWAS) (Option 29) in the current version of the Mendel software is an optimized subroutine for performing large-scale genome-wide quantitative trait locus (QTL) analysis. This analysis (a) works for random sample data, pedigree data, or a mix of both; (b) is highly efficient in both run time and memory requirement; (c) accommodates both univariate and multivariate traits; (d) works for autosomal and x-linked loci; (e) correctly deals with missing data in traits, covariates, and genotypes; ( $f$ ) allows for covariate adjustment and constraints among parameters; (g) uses either theoretical or single nucleotide polymorphism (SNP)-based empirical kinship matrix for additive polygenic effects; (h) allows extra variance components such as dominant polygenic effects and household effects; (i) detects and reports outlier individuals and pedigrees; and (j) allows for robust estimation via the t-distribution. This paper assesses these capabilities on the genetics analysis workshop 19 (GAW19) sequencing data. We analyzed simulated and real phenotypes for both family and random sample data sets. For instance, when jointly testing the 8 longitudinally measured systolic blood pressure and diastolic blood pressure traits, it takes Mendel 78 min on a standard laptop computer to read, quality check, and analyze a data set with 849 individuals and 8.3 million SNPs. Genome-wide expression QTL analysis of 20,643 expression traits on 641 individuals with 8.3 million SNPs takes $30 \mathrm{~h}$ using 20 parallel runs on a cluster. Mendel is freely available at http://www.genetics.ucla.edu/software.
\end{abstract}

\section{Background}

The classical variance component model has been a powerful tool for mapping quantitative trait loci (QTLs) in pedigrees. Polygenic effects are effectively modeled by introducing an additive genetic variance component operating on the kinship coefficient matrix. With unknown or dubious pedigree structure, global kinship coefficients can be accurately estimated from dense markers using either the genetic relationship matrix (GRM) or the method of moments. In GWAS (genome-wide association studies), the 2 alleles of a SNP (single nucleotide polymorphism) shift trait means and can be tested as a fixed effect. However, fitting a variance component model is computationally challenging, especially when it has to be done for a large number of markers. In the

\footnotetext{
*Correspondence: huazhou@ucla.edu

'Department of Biostatistics, University of California, Los Angeles, CA 90095, USA

Full list of author information is available at the end of the article
}

newly released version of the Mendel software [1], Option 29 implements an ultrafast score test for pedigree GWAS. Score tests require no additional iteration under the alternative model. Only SNPs with the most promising score-test $p$ values are further subject to likelihood ratio testing, thus achieving a good compromise between speed and power for large-scale QTL analysis. In this paper, we demonstrate the capabilities of Mendel on the Genetic Analysis Workshop 19 (GAW19) sequencing data.

\section{Methods}

QTL association mapping typically invokes the multivariate normal distribution to model the observed $T$ variate trait $Y \in \mathbb{R}^{n \times T}$ over a pedigree of $n$ individuals. The standard model [2] collects the means of the responses $\operatorname{vec}(\boldsymbol{Y})$ into a vector $\boldsymbol{v}$ and the corresponding 
covariances into a matrix $\boldsymbol{\Sigma}$ and represents the loglikelihood of a pedigree as

$$
L=-\frac{1}{2} \ln \operatorname{det} \boldsymbol{\Sigma}-\frac{1}{2}[\operatorname{vec}(\boldsymbol{Y})-\boldsymbol{v}]^{t} \boldsymbol{\Sigma}^{-1}[\operatorname{vec}(\boldsymbol{Y})-\boldsymbol{v}],
$$

where the covariance matrix is typically parametrized as $\boldsymbol{\Sigma}=2 \boldsymbol{\Sigma}_{a} \otimes \boldsymbol{\Phi}+\boldsymbol{\Sigma}_{d} \otimes \boldsymbol{\Delta}_{7}+\boldsymbol{\Sigma}_{h} \otimes \boldsymbol{H}+\boldsymbol{\Sigma}_{e} \otimes \boldsymbol{I}$. Here $\boldsymbol{\Phi}$ is the global kinship matrix capturing additive polygenic effects, and $\Delta_{7}$ is a condensed identity coefficient matrix capturing dominance genetic effects. For $\boldsymbol{\Phi}$, Mendel can use (a) the theoretical kinship matrix from provided pedigree structures; (b) SNP-based estimates for the kinship of pairs of people within each pedigree; or (c) SNP-based estimates for the entire global kinship matrix ignoring pedigree information. To estimate kinship coefficients from dense SNP data, Mendel employs either the GRM or the method of moments [3, 4]. The household effect matrix $\boldsymbol{H}$ has entries $h_{i j}=1$ if individuals $i$ and $j$ are in the same household and 0 otherwise. Individual environmental contributions and trait measurement errors are incorporated via the identity matrix $\boldsymbol{I}$. QTL fixed effects are captured through the mean component $\boldsymbol{v}=\boldsymbol{A} \boldsymbol{\beta}$ for some predictor matrix $\boldsymbol{A}$ and vector of regression coefficients $\boldsymbol{\beta}$. To test a SNP against a $T$ variate trait, $\boldsymbol{A}$ is augmented with $T$ extra columns holding the allele counts at the SNP, and the corresponding regression coefficients are jointly tested for association [5]. For longitudinal measurements of covariates such as smoke, age, and blood pressure medication (BPmed), we may either assume time varying effect sizes or constrain their effect sizes at different time points to be the same. The latter tactic leads to a more parsimonious and interpretable model and can be easily enforced by setting appropriate parameter constraints in Mendel's control file, which lists the user's choice of model parameters. In Mendel, SNPs with the most impressive test score $p$ values (top 10 by default) are further tested by the more accurate, but slower, likelihood ratio method, thus achieving a good compromise between speed and power for large-scale QTL analysis. We refer readers to our companion manuscript [6] for more model and implementation details.

\section{Results and discussion Family data \\ Size and power study using simulated traits (SIMPHEN.1-200)}

The power to detect the 6 functional variants in the MAP4 gene on chromosome 3 is evaluated from the 200 simulation replicates of the trivariate traits systolic blood pressure (SBP) and diastolic blood pressure (DBP). Type I errors are evaluated from the univariate Q1 trait, which does not involve a major gene. Our analysis includes covariates sex, age, BPmed, smoke, and their pairwise interactions, and uses the theoretical kinship matrix as the additive polygenic variance component. We constrain the covariate effects to be equal across 3 time points. Table 1 shows that the type I error is well controlled. Not surprisingly the power for detecting the 2 rare functional variants $3-47913455$ and $3-47957741$ is extremely low.

\section{QTL analysis of the real, 8-variate phenotype $\left(D B P_{i}, S B P_{i}, i=1,2,3,4\right)$}

Our analyses are based on the genotype calls for 959 individuals (464 directly sequenced and the rest imputed) provided in the chrNN-geno.csv.gz files. SBPs and DBPs measured at 4 time points are available for $1389 \mathrm{mem}$ bers from 20 extended families. The largest family contains 107 individuals; the smallest, 27. Genotypes at $8,348,674$ SNPs were available on 959 of the individuals. We analyzed all SNPs and pedigrees together for the 8 -variate trait $\left(\mathrm{SBP}_{i}, \mathrm{DBP}_{i}, i=1,2,3,4\right)$. Our model includes covariates sex, age, BPmed, smoke, and their pairwise interactions, and we constrain the covariate effects to be equal across 4 time points. The log-likelihoods of the null model (no SNPs included) using the theoretical kinship, GRM within pedigrees, or GRM across all individuals are $-11675.95,-11696.90$, and -11698.71 , respectively, indicating that the provided pedigree information captures additive genetic effects adequately. The

Table 1 Empirical power for testing trivariate DBP and SBP traits and empirical type I error for testing the univariate Q1, based on simulation data in files SIMPHEN.1-SIMPHEN.200

\begin{tabular}{|c|c|c|c|c|c|c|c|c|}
\hline \multirow[t]{2}{*}{ SNP } & \multirow[t]{2}{*}{ MAF } & \multicolumn{3}{|c|}{$\left(\mathrm{DBP}_{1}, \mathrm{DBP}_{2}, \mathrm{DBP}_{3}\right)$} & \multicolumn{3}{|c|}{$\left(\mathrm{SBP}_{1}, \mathrm{SBP}_{2}, \mathrm{SBP}_{3}\right)$} & \multirow{2}{*}{$\begin{array}{l}\mathrm{Q}_{1} \\
\text { Size }\end{array}$} \\
\hline & & $\overline{\beta_{\mathrm{DBP}}}$ & $\% \operatorname{Var}$ & Power & $\beta_{\mathrm{SBP}}$ & $\% \operatorname{Var}$ & Power & \\
\hline $3-47913455$ & 0.0049 & -5.4633 & 0.0036 & $0.05 \pm 0.02$ & -8.7001 & 0.0044 & $0.06 \pm 0.02$ & $0.06 \pm 0.02$ \\
\hline $3-47956424$ & 0.3777 & -1.4951 & 0.0117 & $0.35 \pm 0.03$ & -2.3810 & 0.0143 & $0.42 \pm 0.03$ & $0.03 \pm 0.01$ \\
\hline $3-47957741$ & 0.0016 & -5.0841 & 0.0024 & $0.04 \pm 0.01$ & -8.0964 & 0.0030 & $0.06 \pm 0.02$ & $0.06 \pm 0.02$ \\
\hline $3-47957996$ & 0.0301 & -4.6435 & 0.0122 & $0.82 \pm 0.03$ & -7.3946 & 0.0149 & $0.89 \pm 0.02$ & $0.05 \pm 0.01$ \\
\hline $3-48040283$ & 0.0318 & -6.2235 & 0.0229 & $0.84 \pm 0.03$ & -9.9107 & 0.0278 & $0.89 \pm 0.02$ & $0.05 \pm 0.01$ \\
\hline $3-48040284$ & 0.0131 & -6.9531 & 0.0091 & $0.47 \pm 0.04$ & -11.0726 & 0.0111 & $0.56 \pm 0.06$ & $0.04 \pm 0.01$ \\
\hline
\end{tabular}



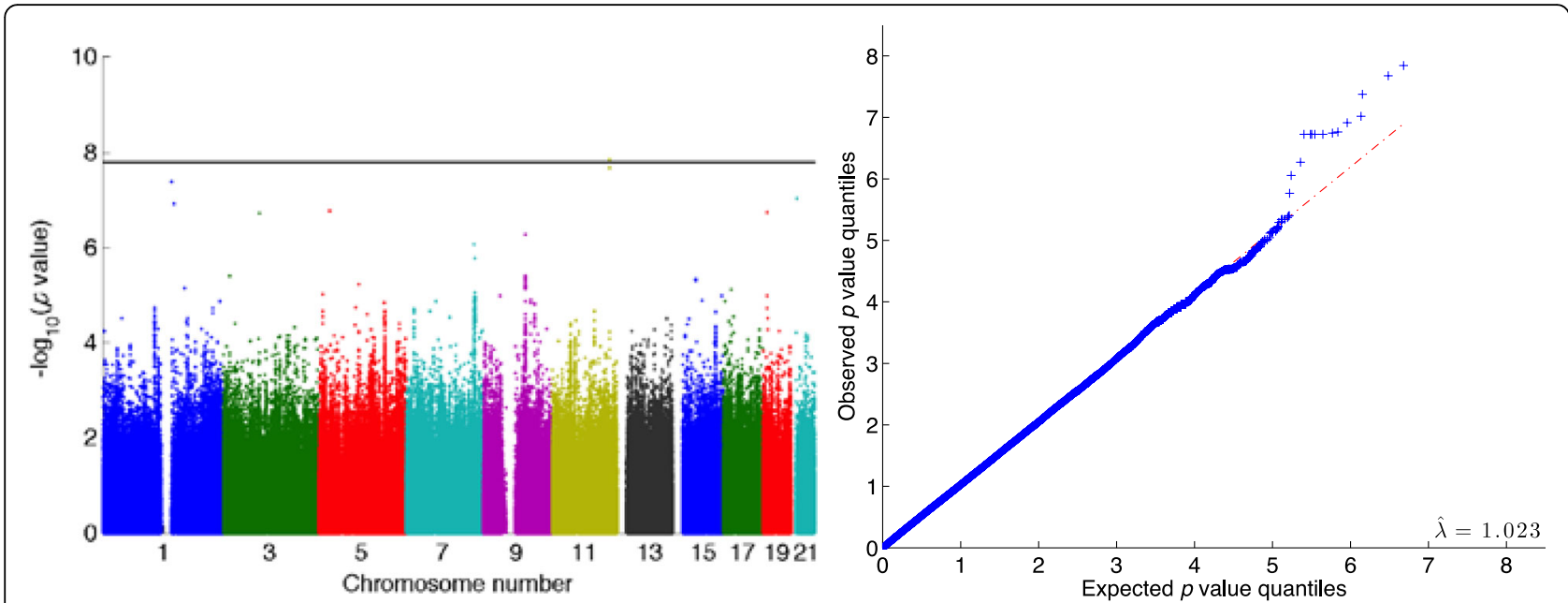

Fig. 1 Multivariate QTL analysis of the real, 8-variate trait $\left(\mathrm{SBP}_{i}, \mathrm{DBP}_{i,} i=1,2,3,4\right)$ from the family data with 849 individuals and 3.1 million SNPs (after filtering). Manhattan plot (left) and quantile-quantile (Q-Q) plot (right). The horizontal line represents the genome-wide significance level. The total run time on a laptop with an Intel Core i7 $2.6 \mathrm{GHz}$ CPU and 16 GB RAM was 78 min

results summarized below use the theoretical kinship matrix.

To read in all the data and run standard quality control (QC) procedures took just under 5 min. QC excluded 10,603 SNPs and 110 individuals based on genotyping success rates below $98 \%$. The remaining $8,338,071$ SNPs and 849 individuals were analyzed. The subsequent ped-GWAS analysis ran in 73 min for all results reported in Fig. 1 and Tables 2 and 3. Because we excluded rare SNPs with low minor allele frequencies $<0.03$ across 849 individuals, $p$ values were calculated for only 3,084,046 SNPs. Accordingly the genome-wide significance threshold is $1.62 \times 10^{-8}$ or 7.79 on the $\log _{10}$ scale; the threshold for a false discovery rate (FDR) of 0.05 is $4.19 \times 10^{-8}$ or 7.38 on the $\log _{10}$ scale.

Table 2 Multivariate QTL analysis of the real, 8-variate trait $\left(\mathrm{SBP}_{i}, \mathrm{DBP}_{i}, i=1,2,3,4\right)$ from the family data with 849 individuals and 3.1 million SNPs (after filtering). Estimated mean effects under the null model (no SNPs included) using the theoretical kinship matrix for the additive polygenic variance component

\begin{tabular}{lrr}
\hline Mean effects & $\mathrm{SBP}_{s i}$ & $\mathrm{DBPs}_{i}$ \\
\hline$\beta_{\text {Sex }}$ & 10.21 & 4.24 \\
$\beta_{\text {Age }_{i}}$ & 0.32 & 0.02 \\
$\beta_{\text {BPmed }_{i}}$ & 3.11 & 10.07 \\
$\beta_{\text {Smoke }_{i}}$ & 1.53 & 1.84 \\
$\beta_{\text {Sex }_{i} \times \text { BPmed }_{i}}$ & -3.20 & -1.84 \\
$\beta_{\text {Sex }_{i} \times \text { Smoke }_{i}}$ & 0.30 & -0.73 \\
$\beta_{\text {Sex }_{i} \times \text { Age }_{i}}$ & 0.41 & 0.14 \\
$\beta_{\text {BPmed }_{i} \times \text { Smoke }_{i}}$ & 3.83 & 2.43 \\
$\beta_{\text {BPmed }_{i} \times \text { Age }_{i}}$ & 0.01 & -0.35 \\
$\beta_{\text {Smoke }_{i} \times \text { Age }_{i}}$ & -0.06 & -0.06 \\
\hline
\end{tabular}

Table 2 lists the estimates for environmental effects and their interactions under the null model (no SNPs included). Figure 1 displays the Manhattan and quantilequantile $(\mathrm{Q}-\mathrm{Q})$ plots. The genomic inflation factor of 1.023 indicates no systematic bias. One SNP passes the Bonferroni-corrected genome-wide significance level, and 3 SNPs pass the FDR 0.05 threshold. They are listed in Table 3. SNP 1-142617328 has a Hardy-Weinberg equilibrium (in founders) $p<10^{-22}$, indicating possible genotyping error. The remaining 2 significant SNPs occur at $118,783,424$ and $118,767,564$ base pairs, respectively, on chromosome 11. Both show a minor allele frequency (MAF) of 0.02778 in 413 founders. Because the MAFs in all 849 individuals are higher than 0.03 , they were not removed in the filtering stage.

\section{Genome-wide expression QTL analysis of 20,634 expression traits}

Genome-wide measures of 20,634 gene expression levels in peripheral blood mononuclear cells from the first study examination are provided for 643 individuals in the family data. The formidable task of exhaustive

Table 3 Multivariate QTL analysis of the real, 8-variate trait $\left(\mathrm{SBP}_{i}, \mathrm{DBP}_{i}, i=1,2,3,4\right)$ from the family data with 849 individuals and 3.1 million SNPs (after filtering). Three SNPs pass the FDR 0.05 threshold. The top SNP, 11-118783424, also passes the genome-wide significance level

\begin{tabular}{lllcc}
\hline SNP & Base pair & MAF & $-\log _{10}(\mathrm{p})$ & HW $p$ value \\
\hline $11-118783424$ & $118,783,424$ & 0.02778 & 7.84 & 0.7665 \\
$11-118767564$ & $118,767,564$ & 0.02778 & 7.68 & 0.7665 \\
$1-142617328$ & $142,617,328$ & 0.49074 & 7.38 & 0.0000 \\
\hline
\end{tabular}

HW Hardy-Weinberg, MAF minor allele frequency 

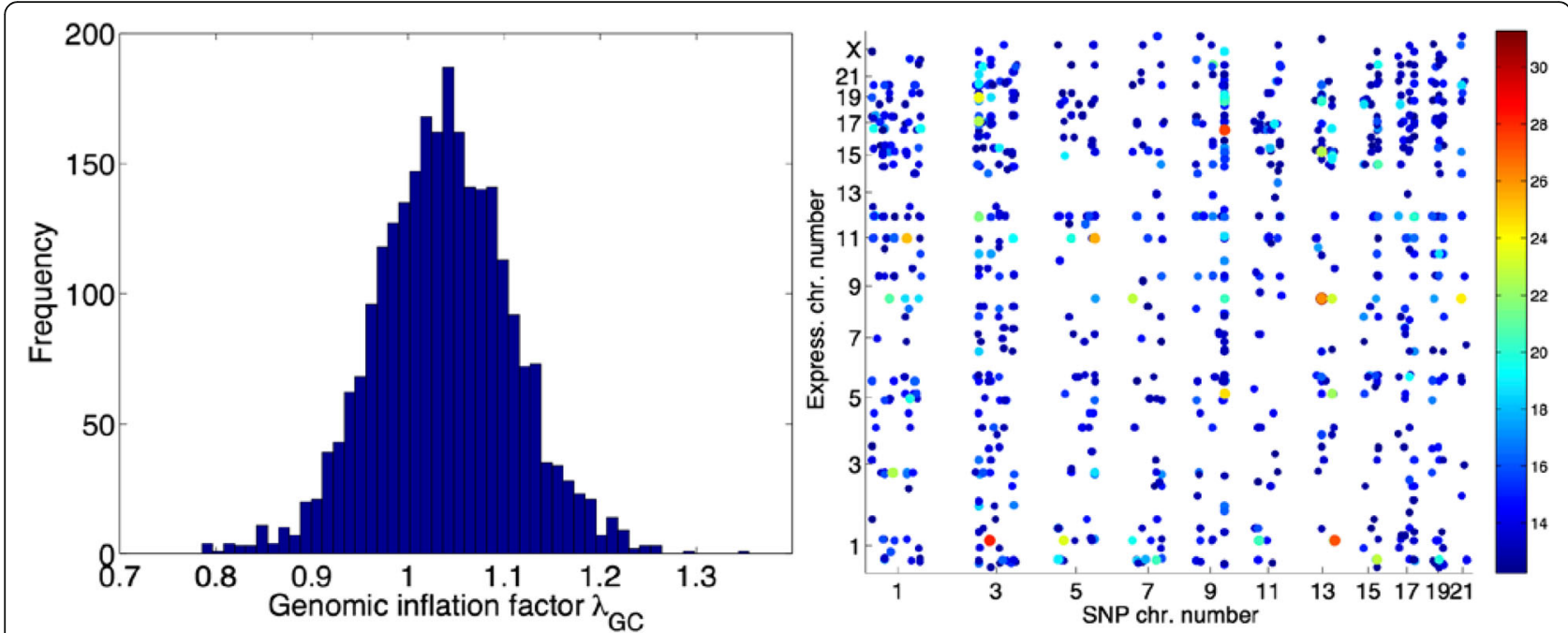

Fig. 2 Summary of the eQTL analysis. Left: Histogram of the genomic inflation factors $\lambda_{G C}$. Right: Top expression-SNP hits from the eQTL analysis. Each dot represents an expression-SNP association that satisfies: genomic inflation factor $\lambda_{G C}<1.1, p<5.77 \times 10^{-13}$, SNP Hardy-Weinberg test (in founders) $p>10^{-8}$, SNP MAF in 641 individuals $>0.01$, and the expression probe is annotated in the EXPR_MAP.csv file. Dot size and color vary according to their $p$ values on the $\log _{10}$ scale. Total run time (20,634 expressions vs. 8,338,071 SNPs) on a cluster with 20 parallel jobs was approximately $30 \mathrm{~h}$

expression quantitative trait locus (eQTL) analysis (20,634 expressions vs. $8,338,071$ SNPs) can be easily managed using Mendel. We submitted 20 parallel jobs to a cluster and finished the complete analysis in approximately $30 \mathrm{~h}$.

In all eQTL runs, SNPs and individuals with genotyping success rate equal to or less than 0.98 are excluded from analysis. Rare variants with MAF equal to orless than 0.01 in all individuals are also excluded. This leaves 641 individuals and 4,199,714 SNPs. The theoretical kinship matrix is used for the additive polygenic variance component. Our analysis includes covariates sex, age, BPmed, smoke, and their pairwise interactions. Initialization takes approximately $5 \mathrm{~min}$; the subsequent genome-wide QTL mapping of each expression trait takes approximately 1 to $2 \mathrm{~min}$. The left panel of Fig. 2 displays a histogram of genomic inflation factors from 20,634 genome-wide QTL analyses. They are well-concentrated

Table 4 Empirical rejection rates (standard errors in parenthesis) for testing five variants in the MAP4 gene against the bivariate (SBP, DBP) trait, based on simulation data in files SIMPHEN.1SIMPHEN.200 for 1943 unrelated individuals

\begin{tabular}{lll}
\hline SNP & MAF & Rejection rate \\
\hline $3-47956424$ & 0.3435 & $1.00(0.00)$ \\
$3-47957741$ & 0.0005 & $0.09(0.02)$ \\
$3-47957996$ & 0.0229 & $1.00(0.00)$ \\
$3-48040283$ & 0.0281 & $1.00(0.00)$ \\
$3-48040284$ & 0.0070 & $0.12(0.02)$ \\
\hline
\end{tabular}

around 1 and indicate no or little systematic bias. The right panel shows the top hits that satisfy a set of stringent criteria listed in the figure caption. Note that the whole eQTL significance level is set at $0.05 / 20634$ / $4199714=5.77 \times 10^{-13}$.

\section{Unrelated data}

A second data set consists of exome sequence calls, blood pressure phenotypes at a single time point, and simulated phenotypes on a large set of unrelated individuals. Like the family data set, these individuals are Mexican Americans; however, they were independently ascertained and do not overlap with the family data set.

\section{Size and power study using simulated traits (SIMPHEN.1-200)}

The data set provides 200 simulation replicates of the trait SBPs and DBP. However, GAW19 organizers did not distribute the exact simulation details, except to state that "The set of causal variants is somewhat different since this is exome data rather than the full sequence data that was provided last time, and so not all of the GAW18 variants, regulatory ones in particular, are present in the new data set." This precludes a precise size and power study.

For ease of comparison, we tested 5 of the 6 variants displayed in Table 1 (for family data) against the bivariate trait (SBP, DBP) for all 200 simulation replicates and report the rejection rates in Table 4. In the model, we include covariates sex, age, BPmed, smoke, and their pairwise interactions, and use the SNP-based 


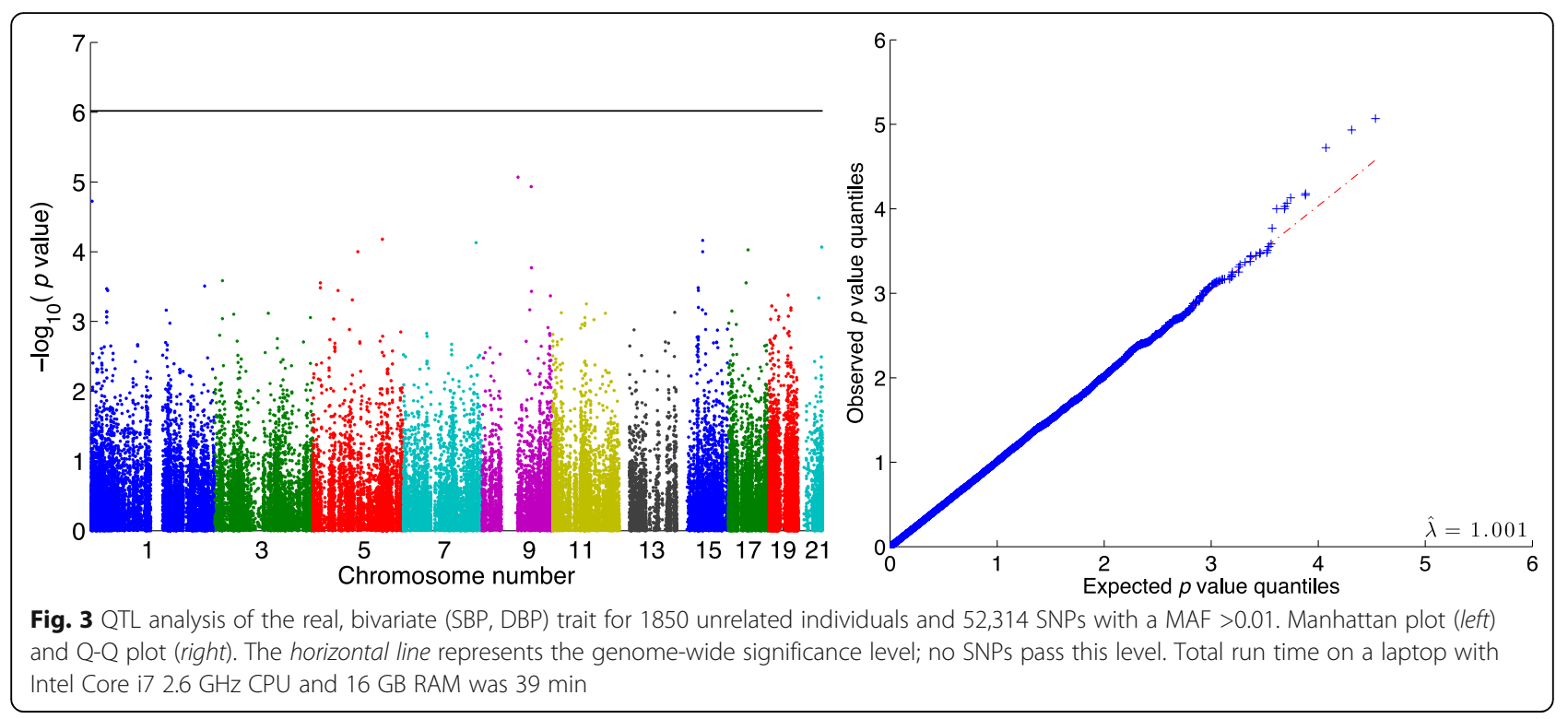

genetic relation matrix for modeling additive polygenic inheritance.

\section{QTL analysis of the real, bivariate phenotypes (DBP and SBP)}

The phenotypes SBP and DBP measured at the first examination are available for 1943 unrelated American Mexicans. We analyzed all SNPs and bivariate traits (SBP, DBP). To read in all the data and run standard QC procedures took $1 \mathrm{~min}$ and $16 \mathrm{~s}$. QC excluded 10,191 SNPs and 93 individuals based on genotyping success rates below $98 \%$. The remaining 1,701,575 SNPs and 1850 individuals were analyzed. GRM calculated from whole genome SNPs was used to adjust for polygenic effects. The subsequent ped-GWAS analysis ran in $37 \mathrm{~min}$ and $5 \mathrm{~s}$ and included all of the results plotted in Fig. 3 and Table 5. Because we exclude rare variants with a MAF equal to or less than 0.01 in all individuals, $p$ values were calculated for 52,314 SNPs. Accordingly, the

Table 5 QTL analysis of the real, bivariate (SBP, DBP) trait for 1850 unrelated individuals and 52,314 SNPs with MAF $>0.01$. Mean effects (standard errors in parenthesis) and variance components under the null model using GRM with all individuals

\begin{tabular}{llll}
\hline Mean effects & SBP & DBP \\
\hline$\mu$ & $94.87(1.62)$ & $78.46(0.95)$ & \\
$\beta_{\text {Sex }}$ & $10.90(1.63)$ & $4.62(0.95)$ & \\
$\beta_{\text {Age }}$ & $0.43(0.05)$ & $-0.13(0.03)$ & \\
$\beta_{\text {Sex } \times \text { age }}$ & $0.38(0.06)$ & & $0.08(0.04)$ \\
Var. comp. & $\Sigma_{a}=\left(\begin{array}{ll}43.15 & 17.03 \\
17.03 & 12.07\end{array}\right)$ & $\Sigma_{e}=\left(\begin{array}{ll}294.88 & 113.90 \\
113.90 & 102.61\end{array}\right)$ \\
\hline
\end{tabular}

genome-wide significance threshold is $9.56 \times 10^{-7}$ or 6.02 on the $\log _{10}$ scale.

Estimated environmental effects and their interactions and variance components under the null model (no SNPs included) are listed in Table 5. Figure 3 displays the Manhattan and Q-Q plots. The genomic inflation factor of 1.001 indicates no systematic bias. No SNPs pass the genome-wide significance level or FDR 0.05 threshold.

\section{Conclusions}

All analyses in this article use Mendel v14.3, which is freely available at http://www.genetics.ucla.edu/software. Pedigree GWAS (Option 29) in Mendel proves to be an extremely efficient and versatile implementation for large-scale QTL analysis. Most competing programs ignore multivariate traits and outliers altogether. See Zhou et al [6] for a side-by-side comparison with the Factored Spectrally Transformed Linear Mixed Model (FaSTLMM) [7] and GEMMA (Genome-wide Efficient MixedModel Analysis) [8] programs. Here we have emphasized Mendel's flexibility in specifying the global kinship matrix, adjusting for confounding, and capturing interactions. These assets, plus its raw speed, make it an ideal environment for QTL mapping. Mendel continues to mature, and geneticists are advised to give it a second look for genetic analysis [1]. In rare variant mapping, each variant may be too rare to achieve significance in hypothesis testing. Grouping related SNPs in a variance component may be more powerful than the mean component models used here. Extending Mendel to test variance component is among the focuses of our current work. 


\section{Acknowledgments}

The authors gratefully acknowledge the National Institutes of Health (NIH) grants GM053275 (EMS and KL) and HG006139 (HZ, EMS, and KL) and National Science Foundation (NSF) grant DMS-1310319 (HZ). The GAW19 whole genome sequence (WGS) data were provided by the T2D-GENES (Type 2 Diabetes Genetic Exploration by Next-generation sequencing in Ethnic Samples) Consortium, which is supported by NIH grants U01 DK085524, U01 DK085584, U01 DK085501, U01 DK085526, and U01 DK085545. The other genetic and phenotypic data for GAW19 were provided by the San Antonio Family Heart Study and San Antonio Family Diabetes/Gallbladder Study, which are supported by NIH grants P01 HL045222, R01 DK047482, and R01 DK053889. The GAW is supported by NIH grant R01 GM031575.

\section{Declarations}

This article has been published as part of BMC Proceedings Volume 10 Supplement 7, 2016: Genetic Analysis Workshop 19: Sequence, Blood Pressure and Expression Data. Summary articles. The full contents of the supplement are available online at http://bmcproc.biomedcentral.com/ articles/supplements/volume-10-supplement-7. Publication of the proceedings of Genetic Analysis Workshop 19 was supported by National Institutes of Health grant R01 GM031575.

\section{Authors' contributions}

$H Z$, EMS, and $K L$ designed the overall study. $H Z$, JZ, and $T H$ conducted statistical analyses. $\mathrm{HZ}$ and JZ drafted the manuscript. All authors read and approved the final manuscript.

\section{Competing interests}

The authors declare they have no competing interests.

\section{Author details}

'Department of Biostatistics, University of California, Los Angeles, CA 90095, USA. ${ }^{2}$ Bioinformatics Research Center, North Carolina State University, Raleigh, NC 27695, USA. ${ }^{3}$ Division of Epidemiology and Biostatistics, Mel and Enid Zuckerman College of Public Health, Tucson, AZ 85721-0066, USA. ${ }^{4}$ Department of Human Genetics, University of California, Los Angeles, CA 90095, USA. ${ }^{5}$ Department of Biomathematics, University of California, Los Angeles, CA 90095, USA. ${ }^{6}$ Department of Statistics, University of California, Los Angeles, CA 90095, USA.

\section{Published: 18 October 2016}

\section{References}

1. Lange K, Papp J, Sinsheimer J, Sripracha R, Zhou H, Sobel EM. Mendel: the Swiss army knife of genetic analysis programs. Bioinformatics. 2013;29:1568-70

2. Lange K. Mathematical and statistical methods for genetic analysis. New York: Springer; 2002.

3. Day-Williams AG, Blangero J, Dyer TD, Lange K, Sobel EM. Linkage analysis without defined pedigrees. Genet Epidemiol. 2011;35(5):360-70.

4. Lange K, Papp JC, Sinsheimer JS, Sobel EM. Next-generation statistical genetics: modeling, penalization, and optimization in high-dimensional data. Annu Rev Stat Appl. 2014;1(1):279-300

5. Lange K, Sinsheimer JS, Sobel EM. Association testing with Mendel. Genet Epidemiol. 2005;29(1):36-50.

6. Zhou H, Blangero J, Dyer TD, Chan KH, Lange K, Sobel EM. Fast genomewide QTL association mapping on pedigree and population data. Genet Epidemiol. In press.

7. Lippert C, Listgarten J, Liu Y, Kadie CM, Davidson RI, Heckerman D. FaST linear mixed models for genome-wide association studies. Nat Methods. 2011;8(10):833-5.

8. Zhou X, Stephens M. Genome-wide efficient mixed-model analysis for association studies. Nat Genet. 2012;44(7):821-4.

\section{Submit your next manuscript to BioMed Central and we will help you at every step:}

- We accept pre-submission inquiries

- Our selector tool helps you to find the most relevant journal

- We provide round the clock customer support

- Convenient online submission

- Thorough peer review

- Inclusion in PubMed and all major indexing services

- Maximum visibility for your research

Submit your manuscript at www.biomedcentral.com/submit
Biomed Central 